\title{
Decline and Recurrence of Stroke Consultations during the COVID-19 Pandemic Lockdown Parallels Population Activity Levels
}

\author{
Felix Schlachetzki ${ }^{a}$ Sibylle Wilfling ${ }^{a} \quad$ Nikolai Dominik Hubert $^{b}$ \\ Andrea Wagner $^{\mathrm{a}}$ Roman L. Haberl ${ }^{\mathrm{b}}$ Ralf Andreas Linker ${ }^{\mathrm{a}}$ Gordian Jan Hubert ${ }^{\mathrm{b}}$ \\ aDepartment of Neurology, TEMPiS Telemedical Stroke Center, Bezirksklinikum Regensburg, University of \\ Regensburg, Regensburg, Germany; ${ }^{b}$ Department of Neurology, TEMPiS Telemedical Stroke Center, Academic \\ Teaching Hospital of the University of Munich, München Klinik Harlaching, Munich, Germany
}

\section{Keywords}

COVID-19 $\cdot$ Stroke $\cdot$ Telestroke $\cdot$ Lockdown $\cdot$ Thrombolysis

\begin{abstract}
Background: The COVID-19 pandemic lockdown (CPL) lead to a significant decrease in emergency admissions worldwide. We performed a timely analysis of ischemic stroke (IS) and related consultations using the telestroke TEMPiS "working diagnosis" database prior (PL), within (WL), and after easing (EL) of CPL. Methods: Twelve hospitals were selected and data analyzed regarding IS (including intravenous thrombolysis [intravenous recombinant tissue plasminogen; IV rtPA] and endovascular thrombectomy [EVT]) and related events from February 1 to June 15 during 2017-2020. In addition, we aimed to correlate events to various mobile phone mobility data. Results: Following the significant reduction of IS, IV rtPA, and EVT cases during WL compared to PL in 2020 longitudinally ( $p$ values $<0.048$ ), we observed increasing numbers of consultations, IS, recommendations for EVT, and IV rtPA with the network in EL over WL not reaching PL levels yet. Absolute numbers of all consultations paralleled best to mobility data of public transportation over walking and driving mobility. Conclusions: While the decrease in emergency
\end{abstract}

karger@karger.com www.karger.com/ced

Karger $\stackrel{\text { ' }}{5}$

GOPEN ACCESS
(C) 2021 The Author(s)

Published by S. Karger AG, Basel

This is an Open Access article licensed under the Creative Commons Attribution-NonCommercial-4.0 International License (CC BY-NC) (http://www.karger.com/Services/OpenAccessLicense), applicable to the online version of the article only. Usage and distribution for commercial purposes requires written permission. admissions including stroke during CPL can only be in part attributed by patients not seeking medical attention, stroke awareness in the pandemic, and direct COVID-19 triggered stroke remains of high importance. The number of consultations in TEMPiS during the lockdown parallels best with mobility of public transportation. As a consequence, exposure to common viruses, well-known triggers for acute cerebrovascular events and other diseases, are reduced and may add to the decline in stroke consultations. Further studies comparing national responses toward the course of the COVID-19 pandemic and stroke incidences are needed.

C) 2021 The Author(s)
Published by S. Karger AG, Basel

\section{Introduction}

The current COVID-19 pandemic challenges diagnostics and treatment of patients with a variety of neurological diseases. Infection with SARS-CoV-2 has been linked to stroke in critically ill patients and unusual stroke manifestations, and may be an additional stroke risk factor and predictor for bad outcome [1-8]. A complicating matter is that in this scenario stroke neurologists need to organize rapid specific stroke management of possible 
COVID-19-positive patients from the preclinical setting through the ambulance and emergency system, to stroke units, and in the neuroradiological department, including IV thrombolysis (intravenous recombinant tissue plasminogen; IV rtPA) and endovascular treatment (EVT), and adequate protection of all hospital personnel [9-11]. Other phenomena related to the COVID-19 pandemic are the compiling reports from hospitals around the globe of a significant decline in a large variety of diseases such as stroke, subarachnoid hemorrhage ( $\mathrm{SAH})$, myocardial infarction, acute heart failure, and diverticular disease, amongst others [12-19].

Various groups noted a timely link of declining stroke cases to the beginning of the various nationwide lockdowns [16, 20-24]. Controversy exists whether or not this phenomenon is purely related to changes in stroke awareness due to reduced social contact or reluctance to seek medical treatment in fear of potential COVID-19 infection after years of successful stroke education efforts and changes in hospital transport guidelines. In addition, some of these publications report additionally lower numbers of IV rtPA, EVT, and large ischemic stroke (IS) volumes while others reported unchanged relations of IV rtPA and EVT within the smaller stroke collective $[2,19$, 24-30]. However, the unprecedented decline in a broad variety of vascular diseases including severe IS, SAH, and myocardial infarction needs further analysis.

In a previous analysis of the TEMPiS telestroke working database, we noted a significant decrease of IV rtPA and EVT recommendations within 6 weeks after beginning of the COVID-19 lockdown to parallel activity data from mobile phones [31, 32]. In this study, we follow up on this development in relation to rising of various activity levels after easing of social distancing measures.

\section{Material and Methods}

Data from daily consultations at 12 hospitals without neurology departments in the telestroke network TEMPiS form the basis of this study as previously described [32]. The consultations took place between February 1 and June 15 in the years 2017-2020. All data were pseudonymized. We extracted the actual working diagnoses based on telemedical consultation and neuroimaging results, mainly cerebral computed tomography. The major "working diagnostic groups" of the TEMPiS data were as follows: (1) IS, (2) transient ischemic attack (TIA), (3) intracranial hemorrhage (ICH, including SAH, subdural hematoma and intracerebral bleeding [ICB]), (4) epileptic seizure, (5) migraine, (6) other disorder (including facial palsy, headache, and brain tumor), and (7) all consultations. Furthermore, cases were analyzed regarding recommendations for IV thrombolysis (IV rtPA) or endovascular therapy (EVT, thrombectomy) for large-vessel occlusion.
Before comparing 2020 data with the previous years, data from 2017 to 2019 (January-July each) were plotted for all consultations, EVT recommendations, and recommendations for IV thrombolysis in order to get a general overview (Fig. 1 and 2). Overall, the number of consultations has been increasing slightly over the years (presumably based on more and better therapies being available, maybe also due to an increase of the average age in the overall population or other causes), while the number of recommendations for IV thrombolysis seemed to stay roughly constant within the analyzed years. As expected, the numbers of EVT recommendations had been increasing from 2017 to 2020, presumably mainly because this method was established as a standard of care. Due to the small case numbers and weekly fluctuations as well as data being only available for the first 6 months of each year, we decided against correction for these increasing numbers. The lack of correction is unfavorable for our working hypothesis - a decrease of case numbers in 2020 due to the lockdown - and thus should not lead to falsely small significances. For this, when comparing with the previous years, we only analyzed the data regarding potentially significant decreases but not increases.

Three 6-week periods were analyzed: prior to (PL) the COVID-19 pandemic lockdown (CPL) from February 1 to March 15, within (WL) the CPL from March 16 to April 30 and upon easing (EL) of the CPL starting May 1 to June 15. These 6-week blocks were divided into 3 subgroups again, each spanning a time frame of 2 weeks. We decided for blocks spanning such small time frames in order to adjust for weekly/seasonal and other fluctuations within the data. Data from the respective time frames from 2017 to 2019 served as reference.

Exploratory descriptive summary statistics with mean values and standard deviations were applied to generate heat maps with intensities defined by the ratio of cases in 2020 divided by the mean number of cases from 2017 to 2019. For better comparability, the overall means and standard deviations of the previous years (January 1-July 15) are shown in an extra column at the right end of the heat map.

Three different analyses of the time periods were applied. In a first step, data from 2020 were compared to data from 2017 to 2019. One sample, two-tailed $t$ tests were used on all subgroups to calculate the corresponding $p$ values. These tests compared the case number in 2020 to the 3 case numbers in 2017, 2018, and 2019. The results are shown as a heat map (Fig. 3) with each field representing one entity in a 2 -week block and the color coding representing the ratio of cases in 2020 to the mean of the case number in the previous 3 years. Red fields depict ratios smaller than 1 - corresponding to a decrease of cases - while blue fields show an increase in cases, which was mainly but not solely to expect for EVT cases in general in 2020.

Additionally, data were analyzed longitudinally in 2020. For this, the data were split into the aforementioned 3 blocks - PL, WL, and EL, each covering 6 weeks - and compared one to another. According to our hypothesis, statistical analysis was performed for the comparison of the lockdown situation with the prior lockdown situation for IS and related events. "ICH" and "Other" events were excluded from this analysis as well as migraine and epilepsy due to relatively small numbers. For clarity reasons, additional data are shown in the heat map, including the development of cases in the EL situation (Fig. 3). As all data are placed within 2020, again there was no correction for increasing or decreasing case numbers under 
Fig. 1. Blot showing the increasing number of recommendations endovascular thrombectomies for the first 6 months from the years 2017 to 2020 . Note the decline with the beginning of the COVID-19 pandemic starting March 2020.
Fig. 2. Blot showing the relatively steady number of recommendations for intravenous thrombolysis for the first 6 months from the years 2017 to 2020 . Note the decline with the beginning of the COVID-19 pandemic starting March 2020.
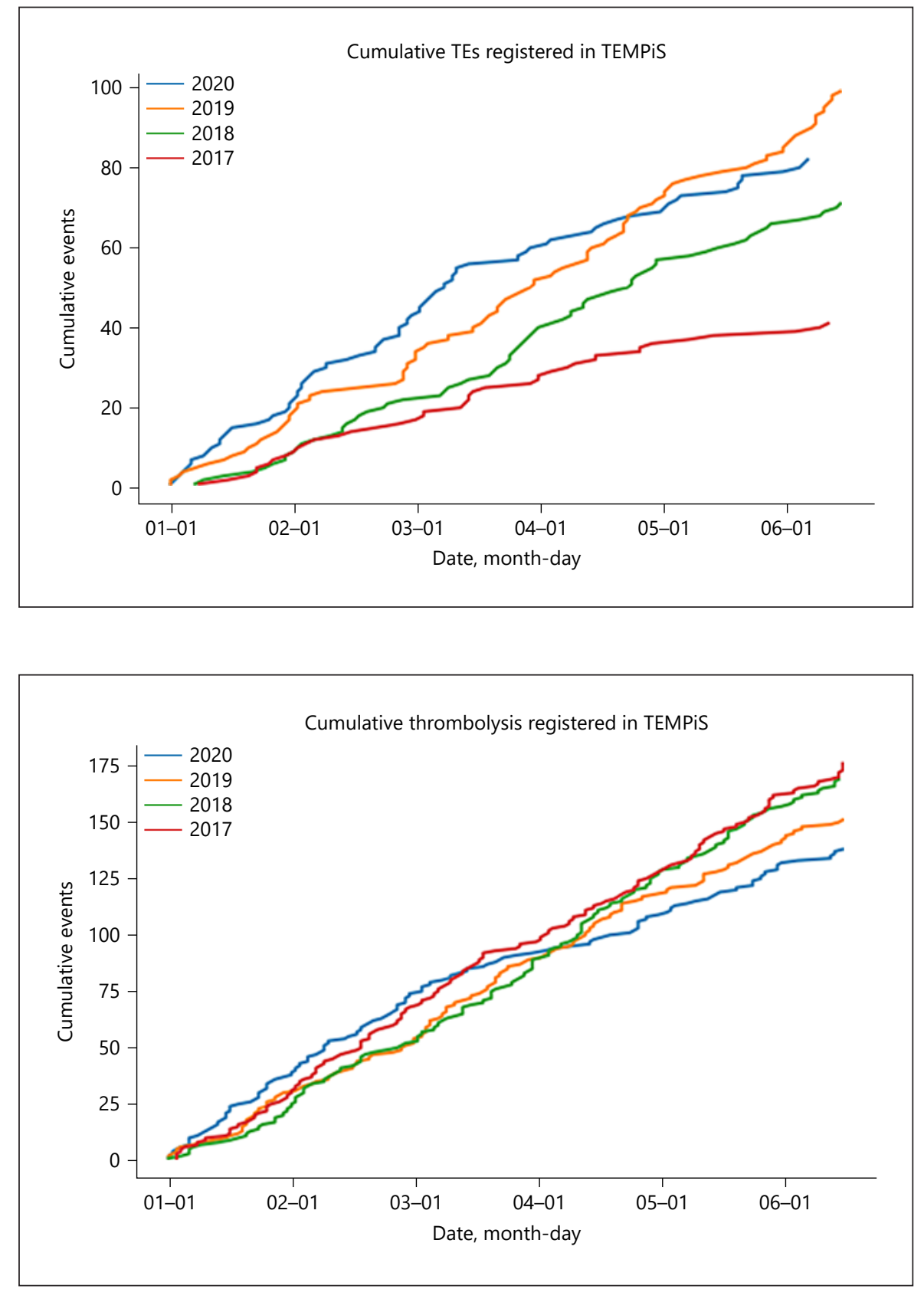

the assumption of minor baseline changes in a time frame of the considered few weeks. As the null hypothesis was strongly onesided - "no change or an increase in cases when comparing the within-lockdown situation to the prior lockdown situation" - onetailed $t$ tests were performed. $p$ values were Bonferroni-corrected, values $<0.05$ were considered significant.

Last, IS and related events information from previous years was combined with longitudinal information. For this, ratios of (cases [2020]/mean [cases 2017-2019]) were compared using one-tailed $t$ tests again with Bonferroni correction and a null hypothesis of an increase or no change in ratios for EL to PL. Statistics were only applied to events that were regarded as virus triggered/IS or isch- emia-related again - so for "all consultations," "EVT recommendations," "IV rtPA recommendations," "IS," and "TIAs".

Furthermore, the heat map shows the number of COVID-positive cases in Bavaria. These data are available for public at https:// experience.arcgis.com/experience/478220a4c454480e823b17327 b2bf1d4/page/page_1/. As these data are given in calendar weeks rather than in daily format, the numbers of COVID-positive cases do not exactly correspond to the given time frames. They only serve as illustration and were not used for any statistical analysis.

General public activity curves related to "walking," "driving," and "public transportation" were created using a moving average over the last 28 days based on the mobility data available at https:// 
Telestroke database working diagnoses 2020

Feb to Jun in comparison to average 2017-2019

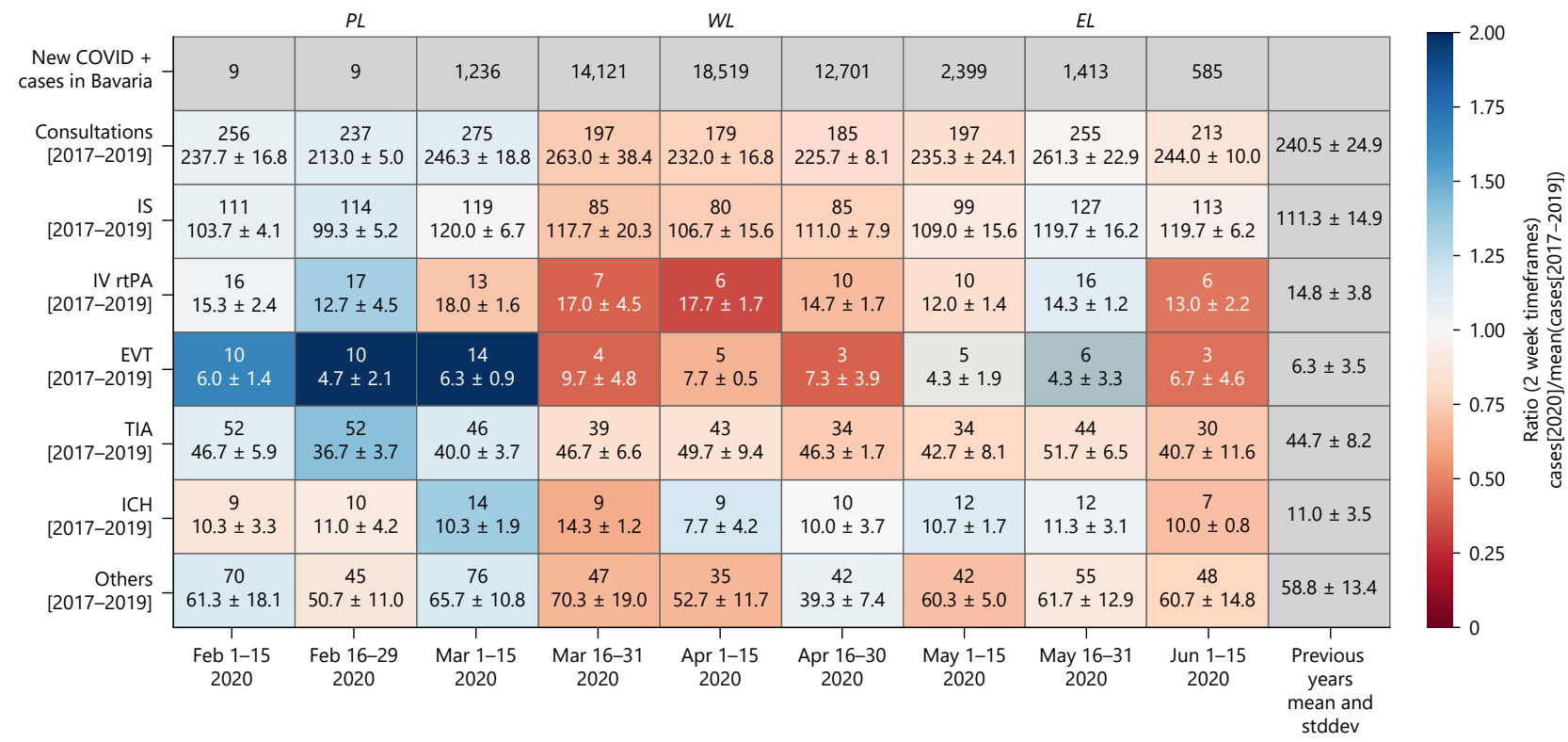

Fig. 3. Heat map of working diagnoses in TEMPiS 2020 compared to 2017-2019.

www.apple.com/covid19/mobility. These mobility data were generated from the relative request volume for directions in specific regions compared to a base volume on January 13,2020. A similar approach was applied for the TEMPiS registered event data before calculating correlations. Traffic data were analyzed for all available regions regarding Bavaria or the whole of Germany - 7 situations with very similar data in total: Munich (driving and walking), Nuremberg (driving, walking, and transit), Bavaria (driving), and Germany (transit). Transit depicts public transportation. Correlations of moving averages of traffic data to IS, IV rtPA, EVT, and consultations in the TEMPiS telestroke database were calculated for each of the 7 available datasets. As all traffic curves showed a very similar course, the data used for our analyses can be regarded as representative for all available datasets in Germany provided by Apple mobility data. As these data include different cities in Bavaria as well as Bavaria and Germany as whole and all the datasets were very similar, it can be assumed to be - at least locally - representative for the TEMPiS data spanning a large area in Bavaria. One might discuss age effects as it is possible that the data given by Apple mobility trends represents a collective younger than the average TEMPiS patient. Due to a lack of representative data, we cannot fully exclude such effects. Nevertheless, we would assume such effects being rather differences in absolute numbers instead of ratios (e.g., less elderly patients being included in Apple mobility trends, but the decline and increase in public transportation, walking and car usage being similar in younger as well as in older patients), thus not affecting the course of the data and consequently also not the correlation.

\section{Results}

A total of 8,498 telemedical consultations with the 12 TEMPiS network hospitals during the specific time frames were investigated. Of these, 29 cases were excluded before further analysis being non-acute consultations (i.e., follow-up examinations). The absolute number of COVID-19-positive cases in the whole of Bavaria rose from a 2- or at maximum small 3-digit area at the end of February 2020 to over 47,000 cases on June 15,2020, with new cases spiking within the COVID-19 lockdown followed by a steady decline thereafter (Fig. 3).

Comparing case numbers in 2020 to the preceding years 2017-2019, no 2-week block reached statistical difference on one-sample, two-tailed $T$ tests when corrected for multiple testing (Fig. 3). The lack of significant changes is due to the small case numbers, high fluctuation in the data, and the triennium serving as reference. Nevertheless, compared to case numbers of previous years, in the weeks prior to lockdown (PL), an increase compared to the case numbers of the previous years indicated in blue heat map colors could be observed, and overall consultations and EVT recommendations were constantly above the means of the previous years. Within the lock- 
Fig. 4. Correlations between events registered in TEMPiS and transit/public transportation in Germany. Shown in the background as semi-transparent are the moving averages of daily case numbers calculated for each day over the last 28 days (IV rtPA, EVT, TIA, IS, and Consultations). Pearson's correlation coefficients are shown as $r$ on the right and were calculated based on unsmoothed averages on a daily basis for a time frame between February 9 and June 15, 2020 (both inclusive). Overlying are smoothed curves for graphical reasons. Values for TIAs are scaled to $1 / 2$, values for IS to $1 / 4$ and values for events overall to $1 / 10$. Transit data are shown as red dashed line. These data are given as the ratio with the denominator (reference) being the value of transit on January 13, 2020. IS, ischemic stroke; IV rtPA, intravenous recombinant tissue plasminogen activator; EVT, endovascular thrombectomy; TIA, transient ischemic attack; ICH, intracranial hemorrhage.

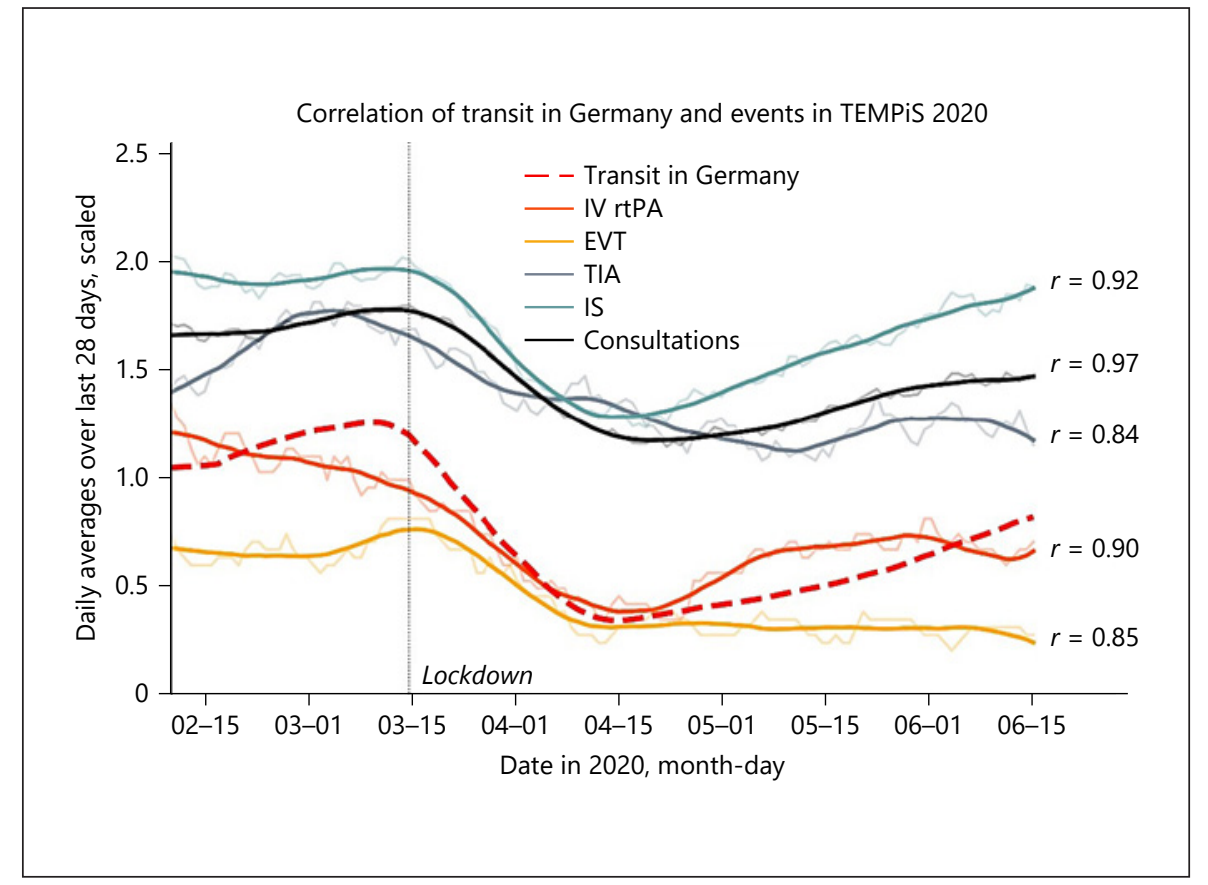

down (WL) phase, the number of consultations, IS, IV rtPA, EVTs, and TIAs stayed constantly below the means of the previous years, indicated in red colors. After the easing of the lockdown (EL phase), a less homogenous picture with some still decreased case numbers compared to the previous years (mainly consultations overall, stroke, and TIA) and other already increasing case numbers could be observed. Overall, this indicates rising case numbers again not reaching PL levels yet.

The analysis of case numbers 6-week blocks in 2020 PL (February 1-March 15), WL (March 16-April 30), and EL (May 1-June 15) revealed significant differences in longitudinal analysis. Comparing the PL to WL period, it exhibited rapid and significant decreases of all consultations, IS, IV rtPA, and EVTs (adjusted $p$ values $<0.0304$, $0.0017,0.0269,0.0472$, and respectively). EL, representing the situation after easing of the lockdown, still revealed decreases.

In order to adjust for both weekly and longitudinal effects, additionally the ratios of (2020/mean [2017-2019]) were compared. Again, the WL ratios of overall consultations, EVTs, and IS showed significant decreases compared to PL (adjusted $p$ values $<0.034$ each). With respect to public mobility indicated by Apple ${ }^{\circledR}$ mobility trends data analysis, the initiation of public lockdown March 15, 2020 , resulted in a decrease in walking, driving, and transit activity in mid-end March 2020 to about $-80 \%$ ( -70.9 to $-82.45 \%$ for 7 different traffic datasets, minimum on
March 21, 2020) on a day-based level, resulting in a decrease to about $40 \%$ (35-45\%) for moving averages over the last 28 days reaching their minima at the midst of April.

When correlating mobility data to telestroke network consultation, correlation coefficients between traffic data and IS ranged from 0.91 to 0.97 (mean 0.94), for IV rtPA from 0.84 to 0.94 (mean 0.90 ), and for EVTs from 0.69 to 0.85 (mean 0.76) for the available datasets. Consultations showed the highest value of correlation with Germany public transport (range $=0.90-0.97$, mean $=0.94$ ), although all mobility datasets showed a similar picture. Specifically, correlation coefficients of Germany public transport and TEMPiS consultations were as followed: 0.97 for all consultations, 0.85 for EVT, 0.90 for rtPA, 0.92 for stroke, and 0.84 for TIAs (Fig. 4). Data of COVIDpositive cases did not parallelize to any of the subgroups of events registered in TEMPiS suggesting no positive correlation.

\section{Discussion}

The current study using the TEMPiS telestroke working database shows a correlation between the decrease and recovery of teleconsultations and population activity levels during COVID-19 lockdown for all stroke subtypes except ICH. The number of consultations with facing dis- 
abling stroke from IS requiring IV rtPA or EVT also diminished and increased in parallel to activity levels without reaching prior levels yet. The COVID-19 lockdown itself appears to have a profound effect not only on stroke occurrence and intensity, but on other (neuro-) vascular diseases and illnesses as well. The common perspective is that altered prehospital awareness and reluctant behavior to seek medical help during the lockdown resulted in diminished and less timely stroke code alerts, yet also different physical activity levels and especially an overall lower risk of general infection due to social distancing and wearing of mouth-nose masks may play a role.

The decline of severe stroke cases requiring IV rtPA and even EVT observed in the TEMPIS telestroke network after initiation of the lockdown parallels the development in a large variety of vascular diseases as shown by several groups [18, 27-29, 32-34]. The general notion in the stroke expert field toward falling numbers of stroke cases strongly suggest that patients and stroke symptoms observing persons refrain to seek medical help because of the risk of SARS-Cov-2 infection in the hospital $[35,36]$. In addition, Aguiar de Sousa et al. [37] speculated on additional reasons for this phenomenon: (1) reduced social contact between relatives may lower third party detection rate of stroke symptoms that patients themselves are often not aware of, (2) the emergency system may be "clotted" with COVID-19 cases and patients do not reach the hospital, (3) ongoing COVID-19 respiratory infection are the leading clinical working diagnosis overshadowing stroke, and (4) altered environmental factors such as reduced pollution may have a more profound effect than previously thought. Indeed, in Akershus University Hospital, Norway, stroke patients during the lockdown had on average a significantly higher NIHSS than before (5.9 vs. 4.2), and upon multivariable logistic regression adjusted for sex, age, living alone, and NIHSS $\leq 5$, there was a significantly increased odds ratio for not reaching hospital within $4.5 \mathrm{~h}$ for IV rtPA [6]. This observation is in line with data from Hongkong, China, with prolonged symptom to hospital time during the CPL [19]. Diegoli et al. [16] analyzing a population-based stroke registry in Joinville, Brazil, found $36.4 \%$ reduction in stroke admissions upon initiation of lockdown measures in 2020 compared to the equivalent time in 2019 with no difference in admissions for severe stroke defined as NIHSS $>8$, ICB, and SAH. These findings may also be local phenomenon as shown by a publication of the Lyon Stroke Center network and the combined descriptive data of 4 German university stroke units by Hoyer et al. [2,38]. The latter group showed a significant decrease in the number of admis- sions for TIA in 3 of 4 centers upon the lockdown by $85 \%$ and a reduction of stroke admission to $46 \%$ in 2 of 4 centers. A relevant effect on reperfusion therapies was found for a single center only with a $60 \%$ reduction for both IV rtPA and EVT. In accordance with our current study they found a positive correlation in 3 of 4 centers between mobility data obtained from Teralytics ${ }^{\circledR}$ (Zurich, Switzerland) and stroke admissions. They conclude that "there are no reasons to assume that the incidence of stroke is decreasing, if anything, it may rise in the context of the pandemic, given the disease-associated predisposition for thromboembolic events." It has to be kept in mind that strict epidemiological data are scarce and travel restrictions, COVID-19 outbreaks in neighboring hospitals with its temporary closure, and re-organization of local emergency pathways to specific high-end hospitals such as university hospitals may limit the assertion of singlecenter studies.

The perhaps largest study to date analyzed data of the central RAPID-software platform and Kansagra and Goyal confirm that also severe stroke volumes diminished during the early lockdown phase of the COVID-19 pandemic [27]. The number of ischemic core volumes $100-150$ and $>150 \mathrm{~mL}$ were observed to decrease by 39.2 and $45.5 \%$, respectively; core volumes $15-100 \mathrm{~mL}$ decreased by 16.6 and $25 \%$; and very small core infarct volumes measuring $0-15 \mathrm{~mL}$ decreased $41 \%$. The decrease in the number of very small infarct volumes may well be explained by the generally proposed hesitation to seek emergency care. However, the observed reduction in large ischemic core volumes, consistent with fewer largevessel occlusions and less severe stroke symptoms, is more in line with our study including the decline in IV thrombolysis and thrombectomy recommendations.

Severe disabling stroke symptoms are impressive events even for non-medical educated persons and initiate emergency alerts, and the same applies to patients experiencing excruciating headache from SAH. Two publications on the rate of SAH during COVID-19 pandemic describe a marked reduction of aneurysmal SAH in Toronto, Canada, and Paris, France $[14,17]$. Our dataset does not show any significant changes in either SAH, ICB, or subdural hematoma; however, the numbers are small. No publications with regard to ICB during the COVID-19 pandemic have been published to the best of our knowledge and the prevalence of ICB in COVID-19 patients appears low [39].

Our data show a parallelization of cerebral ischemic events to traffic data with high correlation coefficients. While correlation cannot prove causality, we suggest that 
this strong correlation may indicate some kind of dependency in between these variables. This, for example, can be observed looking at the number of traumatic brain injuries during the CPL in New York [40]. Another explanation could be, on the other hand side, that there might be one or more other variables influencing both ischemic events and traffic data or that the COVID-19 pandemic event itself influences both the curves without any causality between traffic and the consultation activity in TEMPiS.

Besides the aforementioned changes in prehospital stroke detection, stroke awareness, and avoidance of hospital admission upon only minor stroke symptoms among others during the COVID-19 pandemic, we propose 2 additional mechanisms by which not only stroke but a variety of other diseases may be affected: reduced physical activity - represented by transit data in our study - and low infectious burden by social distancing and face masks. Physical activity is well known to be accompanied by sudden and sometimes extensive blood pressure increases, thus leading to aneurysmal SAH and myocardial infarction $[41,42]$. The most interesting concept for diminishing cardiovascular disease during the COVID-19 pandemic may be an overall reduction of common infection by face masks and social distancing. These measures have recently been proven to be highly effective in reducing the spread of SARS-Cov-2, and may indeed hinder other viral infections $[43,44]$. And general viruses have shown to trigger atherosclerosis and plaque rupture resulting in neuro- and cardiovascular morbidity $[45,46]$. In a nationwide survey in Denmark, Butt et al. [47] also observed falling IS numbers during the CPL but also less cases of influenza virus infection that is well known for triggering IS. A reduction of Helicobacter pylori infection, also a strong risk factor for acute upper gastrointestinal bleeding, may be responsible for an observed reduction of $40 \%$ of this emergency in a majority of Austrian hospitals [48, 49]. Reduced common respiratory tract infection that could trigger acute heart failure and myocardial infarction may explain the up to $50 \%$ decline in cases reported from centers in the USA, Great Britain, Denmark, and France $[15,18,50]$. An interesting recent publication linking reduced stroke incidence and new-onset atrial fibrillation during the COVID-19 pandemic has analyzed the Danish national registry with all of its 5.6 million inhabitants. Holt et al. [50] noted a close to $50 \%$ decrease of new-onset atrial fibrillation following the lockdown, and atrial fibrillation remained strongly associated with severe stroke symptoms, and general inflammation is a strong trigger for atrial fibrillation [51].

Corona, Lockdown, and Stroke
The assumption that additional factors evoked by the COVID-pandemic lockdown such as physical activity and social distancing may lead to a reduced infectious burden and significantly contribute to the decline and now rise in stroke cases in our telestroke network cannot be proven with our dataset. But, the correlation between number of consultations and mobility levels is striking and almost exactly parallels the fast reduction and (slower) recovery. The mobility "walking" may resemble best physical activity, while mobility "transit/ public transportation" may reflect social distancing/face mask wearing as people refrain to public transportation being afraid for SARS-Cov-2 transmission.

Limitations of the study are (a) the retrospective analysis of a teleconsultation registry, not discharge diagnosis, (b) data from 12 hospitals of a larger network were used with a defined structure and defined standard operating procedures not optimized for the COVID-19 pandemic, and (c) incidence was different in each county of the participating hospitals [32]. However, the large amount of data of teleconsultations and the multicenter approach strengthen the results of this study.

\section{Conclusions}

Our study using the TEMPiS telestroke database demonstrate a parallel development of stroke consultations. The magnitude of the decline in IV rtPA and EVT as an indicator for severe stroke as well as reports on reduced SAH and severe cardiac disease by other groups question the notion that only reluctance within the population to seek immediate medical assistance is solely responsible for this phenomenon. We postulate that factors related to mobility, especially reduced physical activity and reduced general infection due to social distancing measures, may significantly contribute as with rising mobility we observe higher stroke consultations in our telestroke network. This effect may potentially complicate stroke treatment with regard to a "second wave." Larger datasets on both, epidemiological data and behavioral changes during the COVID-19 pandemic are needed to shed light into this phenomenon.

\section{Acknowledgements}

The authors acknowledge all consulting neurologists in TEMPiS and colleagues in partner hospitals in Bad Reichenhall, Burglengenfeld, Ebersberg, Eggenfelden, Erding, Freising, Kelheim, Mühldorf, Rotthalmünster, Vilsbiburg, Dingolfing, and Zwiesel.

Cerebrovasc Dis 2021;50:317-325

DOI: $10.1159 / 000514154$ 


\section{Statement of Ethics}

This retrospective study was approved by the local Ethics Committee of the University of Regensburg (20-1789-104) and performed in accordance with guidelines of the World Medical Association Declaration of Helsinki.

\section{Conflict of Interest Statement}

The authors declare no conflict of interest related to this study.

\section{Funding Sources}

The authors did not receive any funding.

\section{Author Contributions}

F.S.: study design and first draft of the manuscript; S.W. and N.D.H.: data analysis and graphical layout; A.W., R.L.H., and R.A.L.: critical revisions of the manuscript; G.J.H.: study design and critical revisions of the manuscript.

\section{References}

1 Belani P, Schefflein J, Kihira S, Rigney B, Delman BN, Mahmoudi K, et al. COVID-19 is an independent risk factor for acute ischemic stroke. AJNR Am J Neuroradiol. 2020 Aug; 41(8):1361-4.

2 Hoyer C, Ebert A, Huttner HB, Puetz V, Kallmünzer B, Barlinn K, et al. Acute stroke in times of the COVID-19 pandemic: a multicenter study. Stroke. 2020 Jul;51(7):2224-7.

3 Mao L, Jin H, Wang M, Hu Y, Chen S, He Q, et al. Neurologic manifestations of hospitalized patients with coronavirus disease 2019 in Wuhan, China. JAMA Neurol. 2020 Jun 1; 77(6):683-90.

4 Miller A, Segan S, Rehmani R, Shabsigh R, Rahme R. Letter: dismantling the apocalypse narrative: the myth of the COVID-19 stroke. Neurosurgery. 2020 Sep 5:nyaa419.

5 Oxley TJ, Mocco J, Majidi S, Kellner CP, Shoirah $\mathrm{H}$, Singh IP, et al. Large-vessel stroke as a presenting feature of Covid-19 in the young. N Engl J Med. 2020 May 14;382(20):e60.

6 Saxhaug Kristoffersen E, Holt Jahr S, Thommessen B, Morten Ronning O. Effect of COVID-19 pandemic on stroke admission rates in a Norwegian population. Acta Neurol Scand. 2020 Dec;142(6):632-6.

7 Yaghi S, Ishida K, Torres J, Mac Grory B, Raz E, Humbert K, et al. SARS-CoV-2 and stroke in a New York healthcare system. Stroke. 2020 Jul;51(7):2002-11.

8 Zhang Y, Xiao M, Zhang S, Xia P, Cao W, Jiang $\mathrm{W}$, et al. Coagulopathy and antiphospholipid antibodies in patients with Covid-19. N Engl J Med. 2020 Apr 23;382(17):e38.

9 Bhaskar S, Sharma D, Walker A. H, McDonald M, Huasen B, Haridas A, et al. Acute neurological care in the COVID-19 era: the Pandemic Health System REsilience PROGRAM (REPROGRAM) Consortium Pathway. Front Neuro. 2020;11:579.

10 Khosravani H, Rajendram P, Notario L, Chapman MG, Menon BK. Protected code stroke: hyperacute stroke management during the coronavirus disease 2019 (COVID-19) pandemic. Stroke. 2020 Jun;51(6):1891-5.
11 Leira EC, Russman AN, Biller J, Brown DL, Bushnell CD, Caso V, et al. Preserving stroke care during the COVID-19 pandemic: potential issues and solutions. Neurology. $2020 \mathrm{Jul}$ 21;95(3):124-33.

12 Andersson C, Gerds T, Fosbøl E, Phelps M, Andersen J, Lamberts $\mathrm{M}$, et al. Incidence of new-onset and worsening heart failure before and after the COVID-19 epidemic lockdown in Denmark: a nationwide cohort study. Circ Heart Fail. 2020 Jun;13(6):e007274.

13 Anteby R, Zager Y, Barash Y, Nadler R, Cordoba M, Klang E, et al. The impact of the coronavirus disease 2019 outbreak on the attendance of patients with surgical complaints at a Tertiary Hospital Emergency Department. J Laparoendosc Adv Surg Tech A. 2020 Sep; 30(9):1001-7.

14 Bernat AL, Giammattei L, Abbritti R, Froelich S. Impact of COVID-19 pandemic on subarachnoid hemorrhage. J Neurosurg Sci. 2020 Aug;64(4):409-10.

15 Bromage DI, Cannata A, Rind IA, Gregorio C, Piper S, Shah AM, et al. The impact of COVID-19 on heart failure hospitalization and management: report from a Heart Failure Unit in London during the peak of the pandemic. Eur J Heart Fail. 2020 Jun;22(6):97884.

16 Diegoli H, Magalhaes PSC, Martins SCO, Moro CHC, Franca PHC, Safanelli J, et al. Decrease in hospital admissions for transient ischemic attack, mild, and moderate stroke during the COVID-19 era. Stroke. 2020 Aug; 51(8):2315-21.

17 Diestro JDB, Li YM, Parra-Farinas C, Sarma D, Bharatha A, Marotta TR, et al. Letter to the editor 'aneurysmal subarachnoid hemorrhage: collateral damage of COVID?' World Neurosurg. 2020 Jul;139:744-5.

18 Hall ME, Vaduganathan M, Khan MS, Papadimitriou L, Long RC, Hernandez GA, et al. Reductions in heart failure hospitalizations during the COVID-19 pandemic. J Card Fail. 2020 Jun;26(6):462-3.

19 Teo KC, Leung WCY, Wong YK, Liu RKC, Chan AHY, Choi OMY, et al. Delays in stroke onset to Hospital arrival time during COVID-19. Stroke. 2020 Jul;51(7):2228-31.
20 Bhatt AS, Moscone A, McElrath EE, Varshney AS, Claggett BL, Bhatt DL, et al. Declines in hospitalizations for acute cardiovascular conditions during the COVID-19 pandemic: a multicenter tertiary care experience. $\mathrm{J}$ Am Coll Cardiol. 2020 Jul 21;76(3):280-8.

21 Bres Bullrich M, Fridman S, Mandzia JL, Mai LM, Khaw A, Vargas Gonzalez JC, et al. COVID-19: stroke admissions, emergency department visits, and prevention clinic referrals. Can J Neurol Sci. 2020 May 26;47(5): 693-6.

22 Morelli N, Rota E, Terracciano C, Immovilli P, Spallazzi M, Colombi D, et al. The baffling case of ischemic stroke disappearance from the Casualty Department in the COVID-19 era. Eur Neurol. 2020;83(2):213-5.

23 Siegler JE, Heslin ME, Thau L, Smith A, Jovin TG. Falling stroke rates during COVID-19 pandemic at a comprehensive stroke center. J Stroke Cerebrovasc Dis. 2020 Aug;29(8): 104953.

24 Uchino K, Kolikonda MK, Brown D, Kovi S, Collins D, Khawaja Z, et al. Decline in stroke presentations during COVID-19 surge. Stroke. 2020 Aug;51(8):2544-7.

25 Esenwa C, Parides MK, Labovitz DL. The effect of COVID-19 on stroke hospitalizations in New York City. J Stroke Cerebrovasc Dis. 2020 Oct;29(10):105114.

26 Kamdar HA, Senay B, Mainali S, Lee V, Gulati DK, Greene-Chandos D, et al. Clinician's perception of practice changes for stroke during the COVID-19 pandemic. J Stroke Cerebrovasc Dis. 2020 Oct:29(10):105179.

27 Kansagra AP, Goyal MS, Hamilton S, Albers GW. Collateral effect of covid-19 on stroke evaluation in the United States. N Engl J Med. 2020 Jul 23;384(4):400-1.

28 Kerleroux B, Fabacher T, Bricout N, Moïse M, Testud B, Vingadassalom S, et al. Mechanical thrombectomy for acute ischemic stroke amid the COVID-19 outbreak: decreased activity, and increased care delays. Stroke. 2020 Jul;51(7):2012-7. 
29 Rudilosso S, Laredo C, Vera V, Vargas M, Renú A, Llull L, et al. Acute stroke care is at risk in the era of COVID-19: experience at a Comprehensive Stroke Center in Barcelona. Stroke. 2020 Jul;51(7):1991-5.

30 Siegler JE, Heslin ME, Thau L, Smith A, Jovin TG. Falling stroke rates during COVID-19 pandemic at a comprehensive stroke center. J Stroke Cerebrovasc Dis. 2020 Aug;29(8): 104953.

31 Audebert HJ, Schenkel J, Heuschmann PU, Bogdahn U, Haberl RL; Telemedic Pilot Project for Integrative Stroke Care G. Effects of the implementation of a telemedical stroke network: the Telemedic Pilot Project for Integrative Stroke Care (TEMPiS) in Bavaria, Germany. Lancet Neurol. 2006 Sep;5(9):7428.

32 Schlachetzki F, Theek C, Hubert ND, Kilic M, Haberl RL, Linker RA, et al. Low stroke incidence in the TEMPiS telestroke network during COVID-19 pandemic - effect of lockdown on thrombolysis and thrombectomy. J Telemed Telecare. 2020 Aug 18.

33 AoK. Available from: https://aok-bv.de/imperia/md/aokbv/aok/zahlen/zuf_2018_ppt_ final.pdf.

34 Amukotuwa SA, Bammer R, Maingard J. Where have our patients gone? The impact of COVID-19 on stroke imaging and intervention at an Australian stroke centre. J Med Imaging Radiat Oncol. 2020 Oct;64(5):607-14.

35 Jasne AS, Chojecka P, Maran I, Mageid R, Eldokmak M, Zhang Q, et al. Stroke code presentations, interventions, and outcomes before and during the COVID-19 pandemic. Stroke. 2020 Sep;51(9):2664-73.

36 Markus HS, Brainin M. COVID-19 and stroke-A global World Stroke Organization perspective. Int J Stroke. 2020 Jun;15(4):3614.
37 Aguiar de Sousa D, Sandset EC, Elkind MSV. The curious case of the missing strokes during the COVID-19 pandemic. Stroke. 2020 Jul; 51(7):1921-3

38 Plumereau C, Cho TH, Buisson M, Amaz C, Cappucci M, Derex L, et al. Effect of the COVID-19 pandemic on acute stroke reperfusion therapy: data from the Lyon Stroke Center Network. J Neurol. 2020 Sep 9.

39 Md Noh MSF. COVID-19 and cerebral hemorrhage: proposed mechanisms. J Neuroradiol. 2020 Jun 1;S0150-9861(20):30194-2.

40 Lara-Reyna J, Yaeger KA, Rossitto CP, Camara D, Wedderburn R, Ghatan S, et al. "Staying home"-early changes in patterns of neurotrauma in New York City during the COVID-19 pandemic. World Neurosurg. 2020 Nov;143:e344-50.

41 Qian Z, Kang H, Tang K, Jiang C, Wu Z, Li Y, et al. Assessment of risk of aneurysmal rupture in patients with normotensives, controlled hypertension, and uncontrolled hypertension. J Stroke Cerebrovasc Dis. 2016 Jul;25(7):1746-52.

42 Kato A, Minami Y, Katsura A, Muramatsu Y, Sato T, Kakizaki R, et al. Physical exertion as a trigger of acute coronary syndrome caused by plaque erosion. J Thromb Thrombolysis. 2020 Apr;49(3):377-85.

43 Chu DK, Akl EA, Duda S, Solo K, Yaacoub S, Schünemann HJ, et al. Physical distancing, face masks, and eye protection to prevent person-to-person transmission of SARS-CoV-2 and COVID-19: a systematic review and meta-analysis. Lancet. 2020 Jun 27;395(10242): 1973-87.
44 Marriott D, Beresford R, Mirdad F, Stark D, Glanville A, Chapman S, et al. Concomitant marked decline in prevalence of SARS-CoV-2 and other respiratory viruses among symptomatic patients following public health interventions in Australia: data from St Vincent's Hospital and associated screening clinics, Sydney, NSW. Clin Infect Dis. 2020 Aug 25.

45 Grau AJ, Urbanek C, Palm F. Common infections and the risk of stroke. Nat Rev Neurol. 2010 Dec;6(12):681-94.

46 Pagliano P, Spera AM, Ascione T, Esposito S. Infections causing stroke or stroke-like syndromes. Infection. 2020 Jun;48(3):323-32.

47 Butt JH, Fosbøl EL, Østergaard L, Yafasova A, Andersson C, Schou M, et al. Effect of COVID-19 on first-time acute stroke and transient ischemic attack admission rates and prognosis in denmark: a nationwide cohort study. Circulation. 2020 Sep 22;142(12): 1227-9.

48 Tielleman T, Bujanda D, Cryer B. Epidemiology and risk factors for upper gastrointestinal bleeding. Gastrointest Endosc Clin N Am. 2015 Jul;25(3):415-28.

49 Schmiderer A, Schwaighofer H, Niederreiter L, Profanter C, Steinle H, Ziachehabi A, et al. Decline in acute upper gastrointestinal bleeding during Covid-19 pandemic after lockdown in Austria. Endoscopy. 2020 Nov; 52(11):1036-8.

50 Holt A, Gislason GH, Schou M, Zareini B, Biering-Sorensen T, Phelps M, et al. New-onset atrial fibrillation: incidence, characteristics, and related events following a national COVID-19 lockdown of 5.6 million people. Eur Heart J. 2020 Jun 1;41(32):3072-9.

$51 \mathrm{Hu}$ YF, Chen YJ, Lin YJ, Chen SA. Inflammation and the pathogenesis of atrial fibrillation. Nat Rev Cardiol. 2015 Apr;12(4):230-43. 